\title{
Complete Resection is Mandatory for Tubercular Cold Abscess of the Chest Wall
}

\author{
Young Tae Kim, MD, PhD, Kook Nam Han, MD, Chang Hyun Kang, MD, PhD, \\ Sook Whan Sung, MD, PhD, and Joo Hyun Kim, MD, PhD \\ Department of Thoracic and Cardiovascular Surgery, Clinical Research Institute, Seoul National University Hospital, Cancer \\ Research Institute, Seoul National University College of Medicine, Seoul, Korea
}

\begin{abstract}
Background. Cold abscess of the chest wall is a rare disease and few literature reports detail any treatment experience with a limited patient number. Hence, an optimal treatment plan remains controversial.

Methods. We retrospectively analyzed patients with cold abscess of the chest wall, focusing on their clinical features, surgical results, and the long-term outcome. Eighty patients were enrolled between May 1981 and April 2005. There were 35 male and 45 female patients, who underwent surgical treatment for cold abscess of the chest wall. The mean age of the patients was $31.4 \pm 12.5$ (14 to 73 ) years. Forty patients $(50.0 \%)$ had previous history of pulmonary tuberculosis. A growing chest wall mass was present in every patient. Surgical treatments performed were as follows: abscess debridement and drainage in $15(18.8 \%)$, complete excision of the abscess without chest wall resection in $9(11.2 \%)$, and complete excision of the abscess including chest wall in 56 patients $(70.0 \%)$.
\end{abstract}

Results. There were no cases of operative mortality. Operative morbidity developed in four patients; two wound infections, one pneumonia, and one prolonged chest tube drainage. Postoperative antituberculous medication was given to all patients. Twelve patients (15.0\%) recurred and required a second operation. The recurrence rate was higher in patients where only drainage of the abscess was performed compared with those in whom complete resection was performed $(40.0 \%$ vs $9.2 \%, p=$ 0.008).

Conclusions. Cold abscess of the chest wall can be surgically managed successfully with low operative risk. Complete resection of the abscess, including a portion of the involved chest wall, is mandatory to avoid recurrence.

(Ann Thorac Surg 2008;85:273-7)

(C) 2008 by The Society of Thoracic Surgeons
A lthough the incidence of tuberculosis has decreased for several decades, it remains as a significant public health problem in several countries. The importance of tuberculosis is increasing in western countries as well, due to an increasing number of immunecompromised patients as well as immigrants from endemic areas. Skeletal tuberculosis accounts for $2.6 \%$ of all tuberculosis cases [1] and chest wall tuberculosis has been reported to be in only $1 \%$ to $10 \%$ of bony tuberculosis cases [2,3]. Chest wall tuberculosis is often not successfully treated by medical therapy and consequently needs a combination treatment of surgery and antituberculous medication $[4,5]$. However, an appropriate treatment strategy remains controversial. While a limited local debridement results in frequent recurrence, which requires repeated surgery, a wide excision including the bony chest wall can result in unwanted extensive operation. Only a few articles have been published regarding the treatment of chest wall cold abscess and the case number of those reports is too small to suggest an appropriate surgical strategy [4-6]. We designed this

\footnotetext{
Accepted for publication Aug 21, 2007.

Address correspondence to Dr Kim, Department of Thoracic and Cardiovascular Surgery, Seoul National University Hospital, Cancer Research Institute, Seoul National University College of Medicine, 28 YongonDong, Chongno-Gu, Seoul, 110-744, South Korea; e-mail: ytkim@snu. ac.kr.
}

study to determine an optimal treatment plan for chest wall cold abscess that focused on the extent of chest wall resection.

\section{Patients and Methods}

We retrospectively reviewed the records of 80 patients who underwent surgical treatment for cold abscess of the chest wall at Seoul National University Hospital during a 23-year period from May 1981 to April 2005. The diagnosis was made based on history taking and physical examinations as well as computed tomography (CT) of the chest (Fig 1). Preoperative bacteriologic diagnosis from a needle aspiration specimen was made in only three patients. The diagnosis of tuberculous cold abscess was confirmed by pathologic examination in all patients (Fig 2). We retrospectively analyzed clinical features and the long-term outcome of the patients focused on the extent of surgical resection and the antituberculous medication given. The patient follow-up information was collected by reviewing medical records as well as searching the National Tuberculosis Registry Data Base. As the hospital should report every case of tuberculosis to the $\mathrm{Na}$ tional Tuberculosis Registry Database in Korea, the database can search tuberculosis patients accurately. If the patient showed no recurrence and the physician decided not to follow-up, and if the patient is not newly listed on 

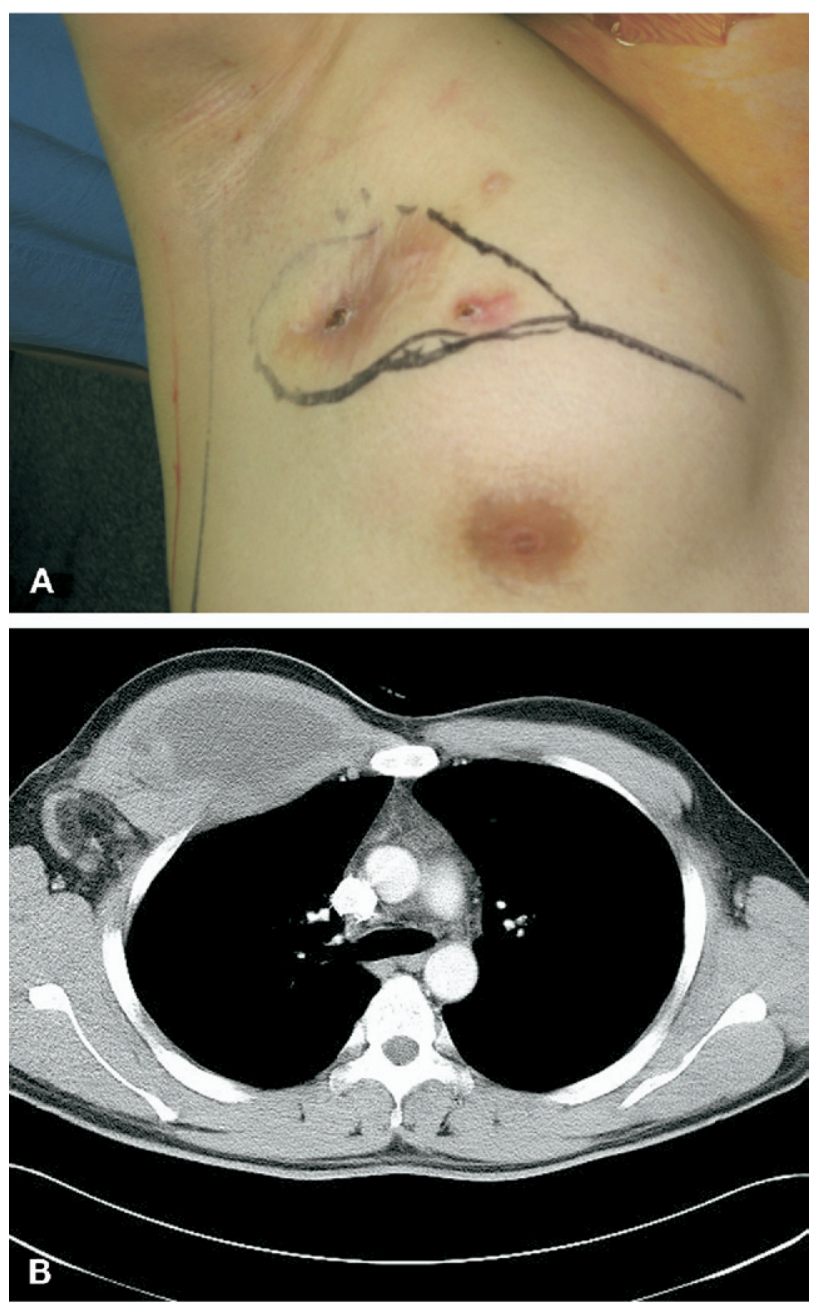

Fig 1. (A) A 27-year-old male patient who presented with a slowly growing soft mass on the right upper anterior chest wall. Note the draining cutaneous fistulae. (B) Computed tomography scan showing a low-attenuate density in the pectoralis major muscles and suggesting chest wall cold abscess.

the National Tuberculosis Database, telephone contacts were attempted. In cases where telephone contact was unsuccessful, the date of last follow-up was used to calculate follow-up duration. Eight patients were lost for follow-up. Patients were divided into two groups based on the extent of resection and the recurrence rates were compared using the $\chi^{2}$ and log-rank tests. Recommendations from the Declaration of Helsinki for Biomedical Research involving Human Subjects were also followed. The study protocol as well as ethical issues were reviewed and approved by the Seoul National University Hospital Institutional Review Board, and the need for patient consent for the study was waived.

\section{Results}

Forty-five patients were women and 35 were men and their mean age at the time of surgery was $31.4 \pm 12.5$ (14 to 73 ) years. All the patients had a growing chest wall mass present. There were no cases of an immune compromised condition. Preoperative bacteriologic culture revealed acid-fast bacilli (AFB) in only three cases. Forty patients $(50.0 \%)$ had either previous or current history of pulmonary tuberculosis and one patient had combined tuberculosis of the spine. Twenty-nine patients $(36.3 \%)$ had been medicated preoperatively with antituberculous chemotherapy for $3.1 \pm 1.8$ (1 to 8$)$ months. Sixteen patients received four-drug therapy with isoniazid, ethambutol, rifampicin, and pyrazinamide, 12 patients received three-drug therapy using isoniazid, ethambutol, and rifampicin, and one patient received cycloserine, prothionamide, aminosalicylic acid, and levofloxacin. The locations of the cold abscess were right chest wall in 51 patients, left chest wall in 14, and anterior chest wall in 15 patients. The timing of the surgery was decided based on the clinical situations such as response to the antituberculosis medication as well as patients' agreement. Surgery was performed between 0 and 8 months $(2.3 \pm$ 1.9 months) after the initial development of the chest wall mass.

Surgical treatments performed were as follows: abscess debridement and drainage in 15 patients (18.8\%), complete excision of the abscess without rib resection in 9 $(11.2 \%)$, and complete excision of the abscess with soft tissue as well as bony chest wall including rib, sternum, or part of clavicle in 56 patients $(70.0 \%)$; right rib in 41 patients, left rib in 13 , a portion of sternum in 4 , and a portion of clavicle in 1. A total of 107 ribs (mean = $1.34 /$ patient) were resected. Soft tissue reconstruction was performed in 14 cases using the latissimus dorsi in eight, the pectoralis major in three, the serratus anterior in one, both latissimus dorsi and serratus anterior in one, and skin graft in one patient. Skeletal reconstruction was performed in four cases; two with GoreTex mesh (W. L. Gore Associates, Flagstaff, AZ), one with Marlex mesh, and one with autologous rib.

The mean hospital stay was 14 days (5 to 60 days). There were no cases of operative mortality. Operative

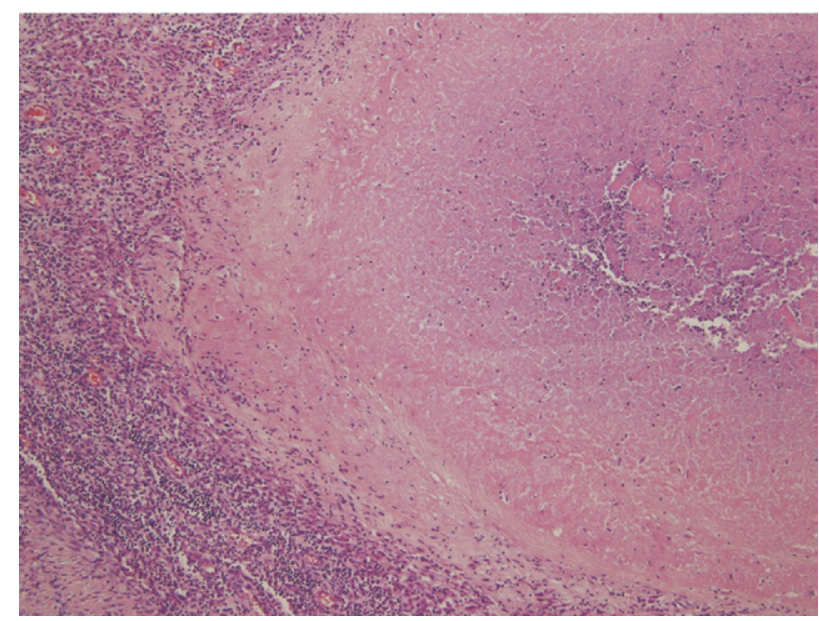

Fig 2. Microscopic finding of the cold abscess showing a typical caseation necrosis with epithelioid histiocyte infiltration. (Hematoxylin and eosin $\times 100$.) 

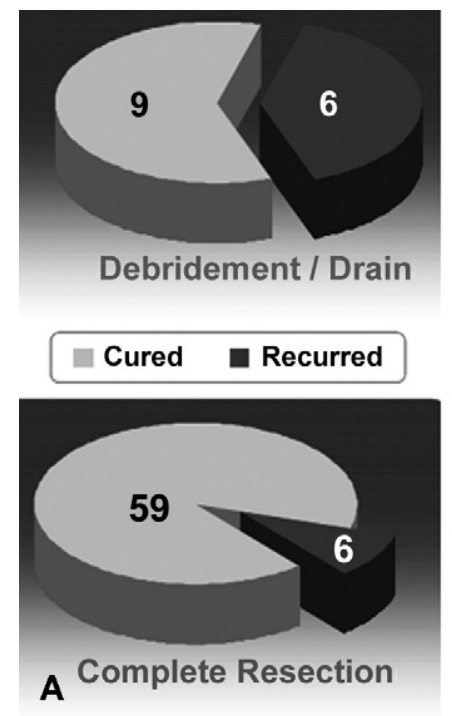

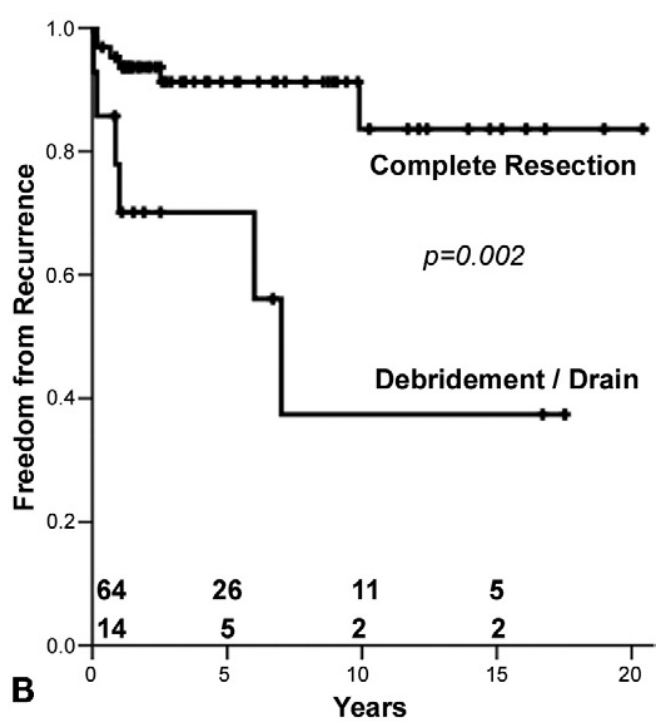

Fig 3. The recurrence according to the surgical procedure performed. (A) The recurrence rates were statistically different $\left(p=0.008, \chi^{2}\right.$ test). (B) The freedom from recurrence after the first operation was superior after compete resection $(p=0.002$, log-rank test $)$. morbidity developed in four patients including two wound infections, one postoperative pneumonia, and one prolonged chest tube drainage. All complications were successfully managed in a conservative manner.

The typical microscopic features with epithelioid histiocytes and caseous necrosis were found in all cases (Fig 2). The AFB were grown in the specimen culture of eight patients. Postoperative antituberculous chemotherapy was given to every patient. Twenty-nine patients were treated for 6 months, 27 for 9 months, 17 for 12 months, and 6 patients received tuberculosis medication for more than one year. The medication given was isoniazid, ethambutol, and rifampicin in 34 patients, isoniazid, ethambutol, rifampicin, and pyrazinamide in 41, isoniazid, ethambutol, rifampicin, and levofloxacillin in 4, and cycloserine, prothionamide, and levofloxacin in one.

During the mean follow-up period of $71.1 \pm 66.9$ (26 to 263) months, eight patients were lost in that time. Twelve patients $(15.0 \%)$ recurred at the same site and required further treatment. Recurrence occurred in six patients $(40.0 \%)$, who were treated with debridement and drainage only, whereas recurrence developed in only six patients $(9.2 \%)$ after wide excision of soft tissue with or without resection of ribs and sternum $\left(p=0.008, \chi^{2}\right.$ test; Fig 3 ). After the debridement and drainage only procedure, recurrence occurred at immediate postoperative period in one patient, at 2 months in one, at 1 year in two, and at 6 years and 7 years in one patient each. Two patients were subsequently treated by wide resection with tuberculous medication and did not report recurrence thereafter. Four patients were treated with repeated drainage and debridement with antituberculosis medication. Among them, one was cured, two recurred after the second operation, and one was lost during follow up without healing of the abscess wound. Six cases of recurrence among patients who received wide complete resection occurred at 2 months in two patients and at 8 months, 1 year, 2.5 years, and 10 years in one patient each. Among them, four patients were treated with a second wide resection and reported no recurrence thereafter. One patient was treated with wound care and was cured. The remaining one patient was treated with antituberculous medication and was lost from follow-up (Table 1).

\section{Comment}

The preoperative diagnosis of chest wall cold abscess has been known to be difficult [6]. Although many authors recommend performing a needle aspiration of the lesion to establish diagnosis, the procedure enables to establish a definite diagnosis in only one-third of the cases [4]. In the area where the prevalence of tuberculosis remains high, the diagnosis can be made based on clinical features such as growing chest wall abscess, as well as history of pulmonary tuberculosis and typical CT findings of a juxtacostal soft tissue mass with central low attenuation and peripheral rim enhancement [7]. Brown [8] suggested making a diagnosis of chest wall cold abscess in Asian or African immigrants who have chest wall abscess and show rib destruction and extrapleural soft tissue masses on CT. The diagnosis of chest wall cold abscess is not difficult in the endemic area of tuberculosis. In Korea, where the prevalence of tuberculosis remains high, we do not routinely recommend needle aspiration to attain a bacteriologic diagnosis unless the clinical features or CT findings suggest the possibility of disease other than cold abscess.

It was interesting that the AFB grew in only eight resected specimens in our series compared to $60 \%$ to $80 \%$ of culture positive results in other studies $[4,5]$. We do not have a clear explanation for this observation. However, recent use of more sensitive diagnostic tools, such as polymerase chain reaction for AFB, may increase the bacteriologic detection rates. We also found nine bacteria or fungus growth in the culture of the resected specimen, including six Staphylococcus species and Pseudomonas, Klebsiella, and Aspergillus species in each one. Among 
Table 1. Outcome of Patients Who Recurred After the First Operation

\begin{tabular}{cllll}
\hline Patient & 1st Operation & Recurred & \multicolumn{1}{c}{ Treatment } & Outcome \\
\hline 1 & Debridement/drain & $<1$ month & Wide resection, Skin graft & Cured \\
2 & Debridement/drain & 2 months & Wound care & Cured \\
3 & Debridement/drain & 1 year & Drain & Recurred \\
4 & Debridement/drain & 1 year & Drain & Recurred \\
5 & Debridement/drain & 6 years & Drain & Lost \\
6 & Debridement/drain & 7 years & Wide resection & Cured \\
7 & Complete resection & 2 months & Wide resection & Cured \\
8 & Complete resection & 2 months & Drain & Cured \\
9 & Complete resection & 8 months & Medication & Lost \\
10 & Complete resection & 1 year & Wide resection & Cured \\
11 & Complete resection & 2.5 years & Wide resection & Cured \\
12 & Complete resection & 10 years & Wide resection & Cured \\
\hline
\end{tabular}

them, Pseudomonas and Klebsiella species were identified in patients whose abscess aggravated to form a cutaneous fistula. We were not able to find any correlation between mixed bacterial infection and clinical outcome. Hence, we presume the Staphylococcus and Aspergillus species could have been contaminants.

It has been reported that the chest wall cold abscess occurs more frequently in men $[4,6,9]$. In our series, however, women were affected more frequently than men. There are different reports with regards to the frequently affected age. While some authors reported more frequent incidence at a younger age ( 15 to 35 years) $[7,9,10]$, others reported a higher rate at an older age $[4,6,11]$. In our current series, $73 \%$ of the patients were younger than 35 years, and only 7 patients $(9 \%)$ were older than 50 years.

There are a few reports $[6,11,12]$ on the successful treatment of chest wall cold abscess with only antituberculous chemotherapy. However, the number of patients in those reports is small and the follow-up was too short. Other authors $[4,5]$ have reported frequent recurrence of cold abscess by treatment with antituberculous medication only. In our experience, 29 patients were treated with antituberculous medication preoperatively for 1 to 8 months. However, the lesion did not heal and eventually needed surgical resection.

Although surgical resection of the abscess usually provides a definite diagnosis and therapy, there are controversies with regard to the treatment approach. Weissberg [13] suggested performing drainage of the abscess for the purpose of tissue diagnosis followed by antimicrobials treatment, reserving debridement and excision only for the most extensive diseases. Sakuraba and colleagues [5] and Faure and colleagues [4] reported that the majority of patients whose abscess were drained first, needed excision for the definite treatment. We also noticed that the cold abscess was rarely treated by antituberculous medication. Although we tried preoperative antituberculous chemotherapy to reduce the size of the abscess and minimize the extent of the chest wall resection, the abscess did not usually respond to the medical treatment, and eventually surgical excision was necessary.
With regard to the extent of chest wall resection, surgical debridement, including resection of the necrotic ribs, cartilages, and visible adenopathies, has been traditionally recommended. However, there is no clear definition of the extent of resection. Previous reports showed a low rate of recurrence. However, the number of patients was too small and the follow-up period was too short [4-6]. Although we experienced slightly high recurrence rates $(13.8 \%)$, it is noteworthy that we experienced recurrence even after 7 to 10 years from the initial operation. Paik and colleagues [14] reported their experience of 89 patients during 30 years and indicated $7.8 \%$ recurrence rates, with one case in which recurrence developed 12 years after surgery. Given the indolent nature of tuberculosis it is important to evaluate the success of treatment by making careful observations for a significant period of time. We classified the surgical method into debridement and drain only and complete resection with or without skeletal resection based on the operation record. We found the recurrence rates were more frequent after debridement and drain only. This result suggests complete resection of the abscess, including all the abscess wall and adjacent bony chest wall, can reduce postoperative recurrence. However, extensive chest wall resection may result in an unnecessary large operative scar or chest wall deformity and sometimes the extent of resection can be compromised. We found no imaging study was helpful in deciding the extent of chest wall resection. The final decision of the resection extent was determined based on the intraoperative finding. We did our best effort to make the extent of resection as small as possible, while removing all the infected tissue. Usually the situation when rib resection was mandatory was when there was a fistulous tract underneath the ribs. Sometimes, the fistulous tract was connected to the lymph nodes located on the inner surface of the chest cage, which supports the hypotheses that the chest wall cold abscess can be caused by direct extension from lymphadenitis of the chest wall [4]. However, we were not able to clearly identify how many patients showed such operative findings in our series. 
A previous report [4] suggested antituberculous medication can prevent long-term recurrence. However, in our experience, all 12 patients who experienced recurrence were treated with postoperative antituberculous medication for longer than 12 months with the exception of two patients who were treated for 2 and 6 months. We agree and recommend treating patients after surgical resection with antituberculous medication. However, it seems that the completeness of the abscess is the more important factor in determining the development of recurrence.

In conclusion, we noticed that complete resection of the cold abscess could be achieved without significant morbidity and could reduce recurrence rates. Based on this result, we recommend that a chest wall cold abscess should be treated by complete resection, including all of the involved area, as well as by the appropriate antituberculous chemotherapy.

\section{References}

1. Enarson DA, Ashley MJ, Grzybowski S, Ostapkowicz E, Dorken E. Non-respiratory tuberculosis in Canada. Epidemiologic and bacteriologic features. Am J Epidemiol 1980;112:341-51.

2. Davies PD, Humphries MJ, Byfield SP, et al. Bone and joint tuberculosis. A survey of notifications in England and Wales. J Bone Joint Surg Br 1984;66:326-30.
3. Newton P, Sharp J, Barnes KL. Bone and joint tuberculosis in Greater Manchester 1969-79. Ann Rheum Dis 1982;41:1-6.

4. Faure E, Souilamas R, Riquet M, et al. Cold abscess of the chest wall: a surgical entity? Ann Thorac Surg 1998;66: 1174-8.

5. Sakuraba M, Sagara Y, Komatsu H. Surgical treatment of tuberculous abscess in the chest wall. Ann Thorac Surg 2005;79:964-7.

6. Hsu HS, Wang LS, Wu YC, Fahn HJ, Huang MH. Management of primary chest wall tuberculosis. Scand J Thorac Cardiovasc Surg 1995;29:119-23.

7. Lee G, Im JG, Kim JS, Kang HS, Han MC. Tuberculosis of the ribs: CT appearance. J Comput Assist Tomogr 1993;17:363-6.

8. Brown TS. Tuberculosis of the ribs. Clin Radiol 1980;31: $681-4$.

9. Tatelman M, Drouillard EJ. Tuberculosis of the ribs. Am J Roentgenol Radium Ther Nucl Med 1953;70:923-35.

10. Brown RB, Trenton J. Chronic abscesses and sinuses of the chest wall; the treatment of costal chondritis and sternal osteomyelitis. Ann Surg 1952;135:44-51.

11. Chen CH, Shih JF, Wang LS, Perng RP. Tuberculous subcutaneous abscess: an analysis of seven cases. Tuber Lung Dis 1996;77:184-7.

12. Blunt SB, Harries MG. Discrete pleural masses without effusion in a young man: an unusual presentation of tuberculosis. Thorax 1989;44:436-7.

13. Weissberg D. Invited commentary: Cold abscess of the chest wall: a surgical entity? Ann Thorac Surg 1998;66:1178.

14. Paik HC, Chung KY, Kang JH, Maeng DH. Surgical treatment of tuberculous cold abscess of the chest wall. Yonsei Med J 2002;43:309-14. 